\title{
The Promotion Rule under Imperfect Observability of the Employee's Ability
}

\author{
Shota Araki', Daiji Kawaguchi'1,2 \\ ${ }^{1}$ Graduate School of Economics, Hitotsubashi University, Tokyo, Japan \\ ${ }^{2} \mathrm{IZA}$, Bonn, Germany \\ Email: ed112001@g.hit-u.ac.jp, kawaguch@econ.hit-u.ac.jp
}

Received 7 July 2014; revised 12 August 2014; accepted 6 September 2014

Copyright (C) 2014 by authors and Scientific Research Publishing Inc.

This work is licensed under the Creative Commons Attribution International License (CC BY).

http://creativecommons.org/licenses/by/4.0/

(c) (i) Open Access

\begin{abstract}
This note provides the closed-form solution for the model by Lazear [1]. The employer adjusts the performance standard for promotion when the employer observes only the imperfect index of the employee's ability. The adjustment margin is larger when the performance depends heavily on luck and depends lightly on the employee's ability.
\end{abstract}

\section{Keywords}

\section{Peter Principle, Promotion, Employer's Learning}

\section{Introduction}

The Peter Principle claims that an employee is promoted to the rank at which the employee exhibits his incompetence. Lazear [1] attributes the observation to the statistical mean reversion. The employer promotes an employee if the employee's performance exceeds a certain threshold. When the employee's performance depends partly on luck, a lucky employee is more likely to be promoted. The promoted employee's performance necessarily declines, on average, because the good luck does not persist after his promotion. Lazear [1] argues that the observed decline has nothing to do with misassignment, because the employer accounts for the mean reversion of the employee's performance when setting the promotion threshold. Lazear [1] qualitatively characterizes the promotion threshold, and provides several numerical examples for this threshold, but does not provide the closed-form solution. This note provides the closed-form solution for the model under normality assumptions on ability and productivity-shock distributions to explicitly demonstrate the model's rich implications.

\section{Setup}

An employer hires an employee whose performance in period $t$ depends on ability $A$ and a random shock 
$\epsilon_{t}$. There are two periods in the production, and there are two types of jobs. Output in period $t$ is $\alpha+\beta\left(A+\epsilon_{t}\right)$ in the easy job and $\gamma+\delta\left(A+\epsilon_{t}\right)$ in the difficult job. Given $\alpha>\gamma$ and $\delta>\beta$, an employer with high productivity has a comparative advantage in the difficult job. If the employer is risk neutral and can observe the employee's ability, the employer assigns the employee with $A>(\alpha-\gamma) /(\delta-\beta)$ to the difficult job. The challenge for the employer is assigning the employee to either a difficult job or an easy job in period two, after observing the noisy measure of ability $A+\epsilon_{1}$ that can be backed out from the first-period output in either job.

The employer knows the probability density function $f(A)$ and $g\left(\epsilon_{t}\right)$. The ability $A$ has a unimodal and symmetric distribution. The productivity shock $\epsilon_{t}$ is independently distributed across periods and symmetrically distributed with a zero mean. With knowledge of the distributions, the employer updates the subjective ability distribution of a specific employee using the error-ridden index of his ability.

The employer promotes the employee if the first-period performance $A+\epsilon_{1}$ exceeds a threshold $A^{*}$. The employer's problem is to set the threshold $A^{*}$ to maximize the expected output:

$$
\begin{aligned}
\Pi\left(A^{*}\right)= & \int_{-\infty}^{\infty} \int_{A^{*}-\epsilon_{1}}^{\infty} \int_{-\infty}^{\infty}\left[\gamma+\delta\left(A+\epsilon_{2}\right)\right] g\left(\epsilon_{2}\right) f(A) g\left(\epsilon_{1}\right) \mathrm{d} \epsilon_{2} \mathrm{~d} A \mathrm{~d} \epsilon_{1} \\
& +\int_{-\infty}^{\infty} \int_{-\infty}^{A^{*}-\epsilon_{1}} \int_{-\infty}^{\infty}\left[\alpha+\beta\left(A+\epsilon_{2}\right)\right] g\left(\epsilon_{2}\right) f(A) g\left(\epsilon_{1}\right) \mathrm{d} \epsilon_{2} \mathrm{~d} A \mathrm{~d} \epsilon_{1} \\
= & \int_{-\infty}^{\infty} \int_{A^{*}-\epsilon_{1}}^{\infty}[\gamma+\delta A] f(A) g\left(\epsilon_{1}\right) \mathrm{d} A \mathrm{~d} \epsilon_{1}+\int_{-\infty}^{\infty} \int_{-\infty}^{A^{*}-\epsilon_{1}}[\alpha+\beta A] f(A) g\left(\epsilon_{1}\right) \mathrm{d} A \mathrm{~d} \epsilon_{1},
\end{aligned}
$$

using the fact that $\epsilon_{2}$ is independent from $A$ and $\epsilon_{1}$ and has a zero mean.

The first-order condition of the output maximization problem is:

$$
\begin{aligned}
\frac{\partial \Pi}{\partial A^{*}} & =\int_{-\infty}^{\infty}-\left[\gamma+\delta\left(A^{*}-\epsilon_{1}\right)\right] f\left(A^{*}-\epsilon_{1}\right) g\left(\epsilon_{1}\right) \mathrm{d} \varepsilon_{1}+\int_{-\infty}^{\infty}\left[\alpha+\beta\left(A^{*}-\epsilon_{1}\right)\right] f\left(A^{*}-\epsilon_{1}\right) g\left(\epsilon_{1}\right) \mathrm{d} \epsilon_{1} \\
& =\int_{-\infty}^{\infty}\left[(\alpha-\gamma)+(\beta-\delta)\left(A^{*}-\epsilon_{1}\right)\right] f\left(A^{*}-\epsilon_{1}\right) g\left(\epsilon_{1}\right) \mathrm{d} \epsilon_{1}=0 .
\end{aligned}
$$

Lazear [1] does not explicitly solve the problem. Instead, he rearranges the first-order condition so that

$$
\begin{aligned}
& {\left[(\alpha-\gamma)+(\beta-\delta) A^{*}\right] \int_{-\infty}^{\infty} f\left(A^{*}-\epsilon_{1}\right) g\left(\epsilon_{1}\right) \mathrm{d} \epsilon_{1}} \\
& \quad=(\beta-\delta) \int_{-\infty}^{\infty} \epsilon_{1} f\left(A^{*}-\epsilon_{1}\right) g\left(\epsilon_{1}\right) \mathrm{d} \epsilon_{1} \\
& \quad=(\beta-\delta) \int_{-\infty}^{0} \epsilon_{1} f\left(A^{*}-\epsilon_{1}\right) g\left(\epsilon_{1}\right) \mathrm{d} \epsilon_{1}+(\beta-\delta) \int_{0}^{\infty} \epsilon_{1} f\left(A^{*}-\epsilon_{1}\right) g\left(\epsilon_{1}\right) \mathrm{d} \epsilon_{1} \\
& \quad=(\beta-\delta) \int_{0}^{\infty} u\left[f\left(A^{*}-u\right)-f\left(A^{*}+u\right)\right] g(u) \mathrm{d} u,
\end{aligned}
$$

by replacing $\epsilon_{1}=-u$ or $\epsilon_{1}=u$. Assuming $f($.$) to be a unimodal and symmetric distribution and that less$ than one half of the employees should be promoted ( $A^{*}$ is above the median of ability distribution),

$$
\begin{gathered}
f\left(A^{*}-u\right)>f\left(A^{*}+u\right) \text {. Then }(\beta-\delta) \int_{0}^{\infty} u\left[f\left(A^{*}-u\right)-f\left(A^{*}+u\right)\right] g(u) \mathrm{d} u>0 \text {, which implies } \\
{\left[(\alpha-\gamma)+(\beta-\delta) A^{*}\right] \int_{-\infty}^{\infty} f\left(A^{*}-\epsilon_{1}\right) g\left(\epsilon_{1}\right) \mathrm{d} \epsilon_{1}>0}
\end{gathered}
$$

Since $f()>$.0 and $g()>0,. A^{*}>(\alpha-\gamma) /(\delta-\beta)$ follows. This is how Lazear [1] shows that the employer inflates the promotion threshold to account for the expected decline after a promotion. He also points to the deflated promotion threshold when more than one half of the employees should be promoted ( $A^{*}$ is below the median of the ability distribution).

\section{The Closed-Form Solution}

We obtain the closed-form solution for the model, assuming $f(A) \sim N\left(\mu, v^{2}\right)$ and $g\left(\epsilon_{t}\right) \sim N\left(0, \sigma^{2}\right)$. With these assumptions, we can rewrite the first-order condition such that 


$$
\begin{aligned}
\int_{-\infty}^{\infty} & {\left[(\alpha-\gamma)+(\beta-\delta)\left(A^{*}-\epsilon_{1}\right)\right] f\left(A^{*}-\epsilon_{1}\right) g\left(\epsilon_{1}\right) \mathrm{d} \epsilon_{1} } \\
& =\int_{-\infty}^{\infty}\left[(\alpha-\gamma)+(\beta-\delta)\left(A^{*}-\epsilon_{1}\right)\right] \frac{1}{\sqrt{2 \pi v^{2}}} \frac{1}{\sqrt{2 \pi \sigma^{2}}} \exp \left(-\frac{\left(A^{*}-\epsilon_{1}-\mu\right)^{2}}{2 v^{2}}-\frac{\epsilon_{1}^{2}}{2 \sigma^{2}}\right) \mathrm{d} \epsilon_{1}=0 .
\end{aligned}
$$

The terms in the exponential function can be decomposed into terms that do not contain the random variable $\epsilon_{1}$ and a term containing it, as follows:

$$
\begin{aligned}
& \exp \left(-\frac{\epsilon_{1}^{2}}{2 \sigma^{2}}-\frac{\left(A^{*}-\epsilon_{1}-\mu\right)^{2}}{2 v^{2}}\right) \\
& \quad=\exp \left(-\frac{\left(A^{*}-\mu\right)^{2}}{2 v^{2}}\right) \exp \left(\frac{\sigma^{2}+v^{2}}{2 \sigma^{2} v^{2}}\left[\frac{\sigma^{2}}{\sigma^{2}+v^{2}}\left(A^{*}-\mu\right)\right]^{2}\right) \exp \left(-\frac{\sigma^{2}+v^{2}}{2 \sigma^{2} v^{2}}\left[\epsilon_{1}-\frac{\sigma^{2}}{\sigma^{2}+v^{2}}\left(A^{*}-\mu\right)\right]^{2}\right) \\
& =\operatorname{Bexp}\left(-\frac{\sigma^{2}+v^{2}}{2 \sigma^{2} v^{2}}\left[\epsilon_{1}-\frac{\sigma^{2}}{\sigma^{2}+v^{2}}\left(A^{*}-\mu\right)\right]^{2}\right),
\end{aligned}
$$

where $B=\exp \left(-\frac{\left(A^{*}-\mu\right)^{2}}{2 v^{2}}\right) \exp \left(\frac{\sigma^{2}+v^{2}}{2 \sigma^{2} v^{2}}\left[\frac{\sigma^{2}}{\sigma^{2}+v^{2}}\left(A^{*}-\mu\right)\right]^{2}\right)$.

Using this result, the first-order condition becomes:

$$
\begin{aligned}
& B \frac{1}{\sqrt{2 \pi v^{2}}} \frac{1}{\sqrt{2 \pi \sigma^{2}}}\left[(\alpha-\gamma)+(\beta-\delta) A^{*}\right] \int_{-\infty}^{\infty} \exp \left(-\frac{\sigma^{2}+v^{2}}{2 \sigma^{2} v^{2}}\left[\epsilon_{1}-\frac{\sigma^{2}}{\sigma^{2}+v^{2}}\left(A^{*}-\mu\right)\right]^{2}\right) \mathrm{d} \epsilon_{1} \\
& +B \frac{1}{\sqrt{2 \pi v^{2}}} \frac{1}{\sqrt{2 \pi \sigma^{2}}}[-(\beta-\delta)] \int_{-\infty}^{\infty} \epsilon_{1} \exp \left(-\frac{\sigma^{2}+v^{2}}{2 \sigma^{2} v^{2}}\left[\epsilon_{1}-\frac{\sigma^{2}}{\sigma^{2}+v^{2}}\left(A^{*}-\mu\right)\right]^{2}\right) \mathrm{d} \epsilon_{1}=0 .
\end{aligned}
$$

By defining $C^{2}=\left(\frac{\sigma^{2}+v^{2}}{\sigma^{2} v^{2}}\right)^{-1}$, the equation becomes

$$
\begin{aligned}
& B \frac{1}{\sqrt{2 \pi v^{2}}} \frac{1}{\sqrt{2 \pi \sigma^{2}}}\left[(\alpha-\gamma)+(\beta-\delta) A^{*}\right] \sqrt{2 \pi C^{2}} \int_{-\infty}^{\infty} \frac{1}{\sqrt{2 \pi C^{2}}} \exp \left(-\frac{1}{2 C^{2}}\left[\epsilon_{1}-\frac{\sigma^{2}}{\sigma^{2}+v^{2}}\left(A^{*}-\mu\right)\right]^{2}\right) \mathrm{d} \epsilon_{1} \\
& +B \frac{1}{\sqrt{2 \pi v^{2}}} \frac{1}{\sqrt{2 \pi \sigma^{2}}}[-(\beta-\delta)] \sqrt{2 \pi C^{2}} \int_{-\infty}^{\infty} \epsilon_{1} \frac{1}{\sqrt{2 \pi C^{2}}} \exp \left(-\frac{1}{2 C^{2}}\left[\epsilon_{1}-\frac{\sigma^{2}}{\sigma^{2}+v^{2}}\left(A^{*}-\mu\right)\right]^{2}\right) \mathrm{d} \epsilon_{1}=0 .
\end{aligned}
$$

Using the facts that the probability-density function of the normal distribution with mean $\frac{\sigma^{2}}{\sigma^{2}+v^{2}}\left(A^{*}-\mu\right)$ and variance $C^{2}$ integrates to 1 and has the expected value $\frac{\sigma^{2}}{\sigma^{2}+v^{2}}\left(A^{*}-\mu\right)$, the first-order condition becomes:

$$
B \frac{1}{\sqrt{2 \pi v^{2}}} \frac{1}{\sqrt{2 \pi \sigma^{2}}}\left[(\alpha-\gamma)+(\beta-\delta) A^{*}\right] \sqrt{2 \pi C^{2}}+B \frac{1}{\sqrt{2 \pi v^{2}}} \frac{1}{\sqrt{2 \pi \sigma^{2}}}[-(\beta-\delta)] \sqrt{2 \pi C^{2}} \frac{\sigma^{2}}{\sigma^{2}+v^{2}}\left(A^{*}-\mu\right)=0
$$

Dividing the first-order condition by common factors renders:

$$
\left[(\alpha-\gamma)+(\beta-\delta) A^{*}\right]=(\beta-\delta) \frac{\sigma^{2}}{\sigma^{2}+v^{2}}\left(A^{*}-\mu\right)
$$


This leads to the solution:

$$
A^{*}=\frac{(\alpha-\gamma)}{(\beta-\delta)}+\frac{\sigma^{2}}{v^{2}}\left[\frac{(\alpha-\gamma)}{(\beta-\delta)}-\mu\right]
$$

\section{Implications}

Lazear [1] provides numerical solutions on page 147 under the normality assumptions on $f(),. g($.$) and the$ parameter values $\alpha=1, \beta=0.5, \gamma=0, \delta=1, \mu=0, v^{2}=1$. For the case of $\sigma^{2}=1$, Lazear's [1] $A^{*}=4.01$ is close to our solution $A^{*}=4$. For the case of $\sigma^{2}=0.1$, Lazear's [1] $A^{*}=2.08$ is again close to our solution $A^{*}=2.2$. The examples make a point that the employer sets a higher threshold if the performance depends heavily on luck, because the employer expects a severe performance decline in the second period.

The closed-form solution preserves the predictions in the original model. In a typical case in which fewer than one half of employees are eligible for promotion, $(\alpha-\gamma) /(\beta-\delta)>\mu$, in order to compensate for the expected decline, the employer sets a higher threshold for promotion than the case when the employer perfectly observes the employee's ability. This threshold premium is larger when the employer knows that the first-period performance depends heavily on luck and depends lightly on ability so that $\sigma^{2} / v^{2}$ is larger. The argument reverses when more than one half should be promoted, $(\alpha-\gamma) /(\delta-\beta)<\mu$. The employer thus discounts the threshold, expecting a future rise of the employee's performance, particularly when the first-period output depends heavily on luck and depends lightly on ability.

\section{Acknowledgments}

This work was supported by JSPS KAKENHI Grant Numbers 23330079 and 11J02356. This support is greatly appreciated.

\section{References}

[1] Lazear, E. (2004) The Peter Principle: A Theory of Decline. Journal of Political Economy, 112, S141-S163. http://dx.doi.org/10.1086/379943 
Scientific Research Publishing (SCIRP) is one of the largest Open Access journal publishers. It is currently publishing more than 200 open access, online, peer-reviewed journals covering a wide range of academic disciplines. SCIRP serves the worldwide academic communities and contributes to the progress and application of science with its publication.

Other selected journals from SCIRP are listed as below. Submit your manuscript to us via either submit@scirp.org or Online Submission Portal.
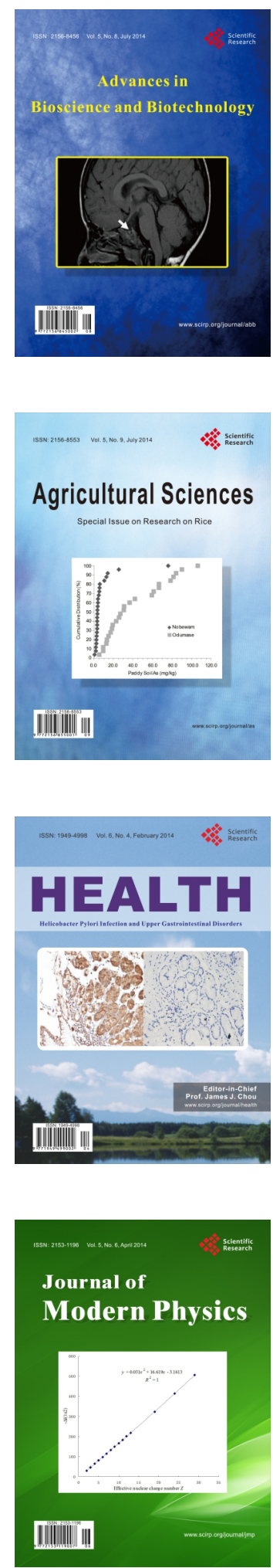
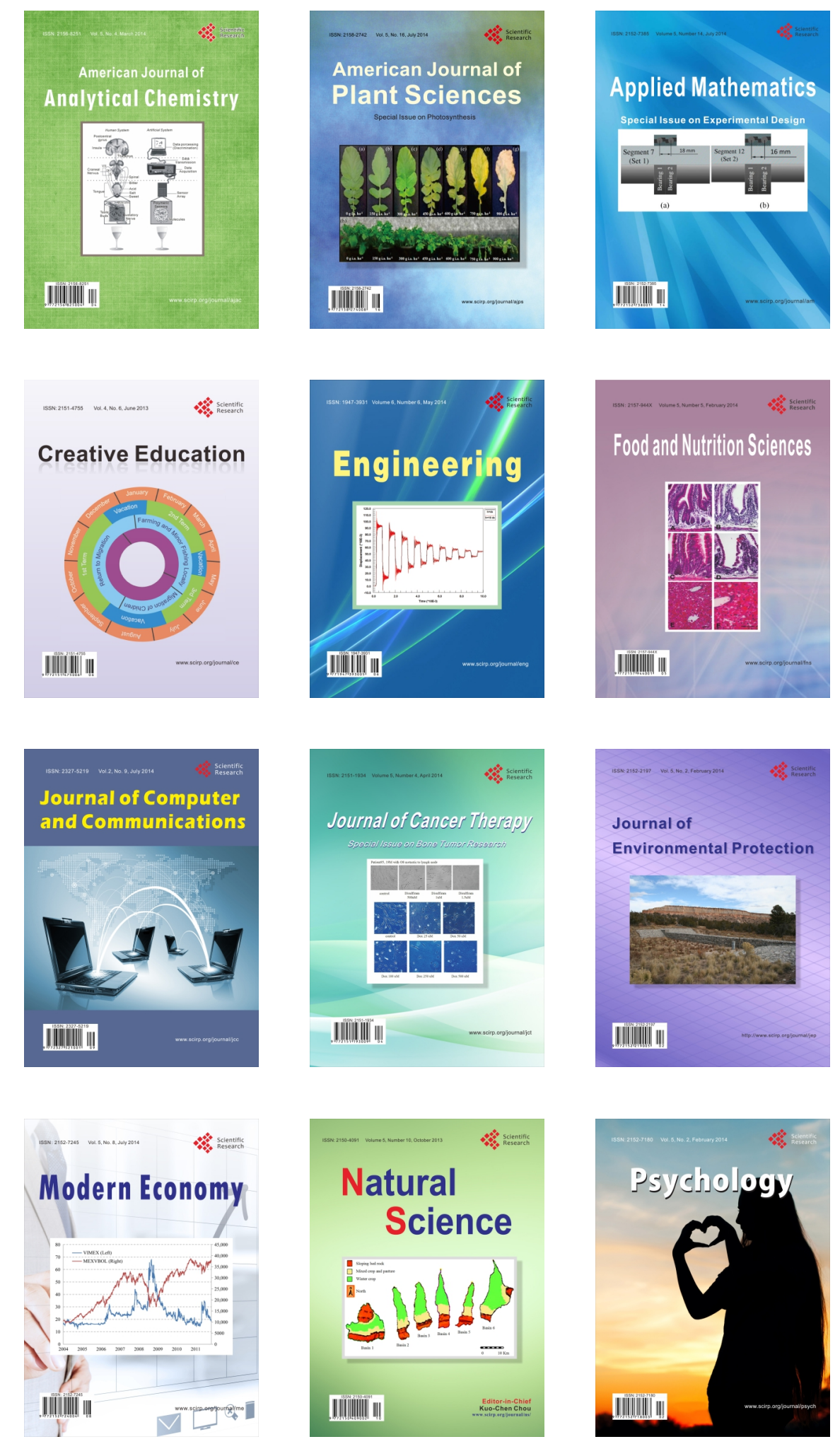